\title{
Hyperglycemic Hyperosmolar State (HHS) with New-onset Diabetes Mellitus in a Patient with SARS CoV-2 Infection.
}

\author{
Awaji Q. Al-Naami ${ }^{1}$, Liaqat Ali Khan ${ }^{1}$, Faisal I. Zaidan ${ }^{1}$, Ibrahim A. Al-Neami ${ }^{1}$, Akram S. \\ Awad $^{1}$, Abdulwahab I. Hobani ${ }^{1}$, Ali H. Sheikh ${ }^{1}$, and Mousa A. Ahmadini ${ }^{1}$ \\ ${ }^{1}$ Affiliation not available
}

November 23, 2020

\begin{abstract}
New-onset DM or unmasking existing one, with or without metabolic complications, has been reported in SARS CoV-2 infection. New-onset DM in association with HHS alone or combination with DKA is uncommon but a possible manifestation of SARS $\mathrm{CoV}-2$ infection that poses management challenges where the outcome may be worst.
\end{abstract}

Hyperglycemic Hyperosmolar State (HHS) with New-onset Diabetes Mellitus in a Patient with SARS CoV-2 Infection.

Awaji Q. Al-Naami, Liaqat A. Khan, Faisal I. Zaidan, Ibrahim A. Al-Neami, Akram S. Awad, Abdulwahab I. Hobani, Ali H. Shaikh, Mousa A. Ahmadini.

Jazan Health, Kingdom of Saudi Arabia.

Corresponding Author :

Liaqat Ali Khan

drliaqatalikhan@yahoo.com

\section{Abstract:}

New-onset DM or unmasking existing one, with or without metabolic complications, has been reported in SARS CoV-2 infection. New-onset DM in association with HHS alone or combination with DKA is uncommon but a possible manifestation of SARS CoV-2 infection that poses management challenges where the outcome may be worst.

Keywords : COVID-19, SARS CoV-2, Infection, Diabetes Mellitus, HHS, DKA.

\section{Introduction :}

The ongoing pandemic of COVID -19, which started in Wuhan, China, in late December 2019, affects millions worldwide so far. As of November 22, 2020, over 58 million people infected over 191 countries/regions ${ }^{1}$ caused by severe acute respiratory syndrome coronavirus-2 (SARS CoV-2).

The more vulnerable individuals are aged, with comorbid conditions like diabetes, hypertension, obesity, and low immunity ${ }^{2,3,4}$. SARS CoV-2 may have a diabetogenic effect probably by direct $\beta$-cell damage that not only worsens pre-existing diabetes but probably accelerate pre-diabetes to established one. Yang et al. explained the $\beta$-cell damage mediated by SARS CoV, a virus of the same family causing insulin-dependent diabetes mellitus as $\beta$-cells carry angiotensin-converting enzyme 2 (ACE2) expression ${ }^{5}$. SARS CoV-2 binds 
and enters human cells via ACE2, a transmembrane glycoprotein ${ }^{6}$ that may dysregulate $\beta$-cell function and consequently diabetes.

Herein we report a SAR CoV-2 positive patient with HHS and new-onset diabetes mellitus (DM) with a fatal outcome.

\section{Case Presentation :}

A 46-year-old male, a teacher by profession, presented to the emergency department with a history of fever, cough, shortness of breath, and irritability for the last week. By inquiring, the patient gives a history of increased thirst and urination for one week. There was no history of any medical illness and within normal ranged laboratory workup during annual screening a month ago. Clinical evolution of the patient shows an ill-looking middle-aged dehydrated irritable patient with a $\mathrm{BMI}$ of 27 , vitals; fever, $39 \mathrm{C}^{0}$ pulse, $91 / \mathrm{min}$, respiratory rate, $28 / \mathrm{min}$, blood pressure $(\mathrm{BP}), 105 / 70 \mathrm{mmHg}, \mathrm{SPO}_{2} 94 \%$ on 5lit oxygen on nasal cannula and decreased air entry in the right lung.

Initial laboratory workup shows, blood sugar level, $36.5 \mathrm{mmol}, \mathrm{pH} 7.4, \mathrm{Na}^{+} 133 \mathrm{mmol} / \mathrm{dL}, \mathrm{K}^{+} 4.1 \mathrm{mmol} / \mathrm{dL}$, $\mathrm{Cl}^{-} 78 \mathrm{mmol} / \mathrm{dL}, \mathrm{HCO}_{3}^{-} 29 \mathrm{mmol} / \mathrm{dL}$, Creatinine $2.15 \mathrm{mg} / \mathrm{dL}, \mathrm{BUN} 52 \mathrm{mg} / \mathrm{dL}$, and serum osmolality $329 \mathrm{mOsmol} / \mathrm{kg}, \mathrm{HbA} 1 \mathrm{c}, 87 \mathrm{mmol} / \mathrm{mol}$. and urine sample was negative for ketones. The patient was managed in ED as per protocol and admitted in intermediate care. Due to the ongoing COVID-19 pandemic and signs \& symptoms suggestive of SARS CoV-2 infection, RT-PCR was requested and came positive a day after. The next day patient's conscious level deteriorated and $\mathrm{SPO}_{2}$ dropped to $90 \%$, thus shifted to the intensive care unit (ICU) and put on mechanical ventilation. On the fifth admission day, the patient's $\mathrm{SPO}_{2}$ dropped to $84 \%$ followed by the arrest that was managed by the resuscitation team but unfortunately the patient not was revived.

\section{Discussion :}

A well-documented factor that precipitates diabetes complications in known or new-onset diabetes is infection. It is not apparent that diabetes mellitus increases the chance of SARS CoV-2 infection, however, it is evident that the outcome of COVID-19 is severe in patients with diabetes mellitus ${ }^{7}$.

COVID -19 and diabetes have a two-way relationship. On one hand, diabetes mellitus relates to more severe COVID -19 through exaggerated pro-inflammatory cytokine response and a downregulated ACE2 levels along with compromised innate immunity in addition to the use of angiotensin-converting enzyme inhibitors and angiotensin-receptor blockers (ACEi/ARBS). On the other hand, COVID -19 worsens the control of glues in diabetic patients probably by direct virus-induced $\beta$-cell damage, insulin resistance, and indirectly via medications ${ }^{8}$.

There are reports of new-onset diabetes in patients with SARS CoV-2 confirmed infections. Tim et al ${ }^{9}$. report a young German boy with new-onset type 1 diabetes mellitus presented with DKA, and a patient with similar findings by Hadil et $\mathrm{al}^{10}$. in a healthy middle-aged man. A case series of three new-onset diabetes in SARS CoV-2 infected patients by Thirunavukkarasu et.al ${ }^{11}$. with the possibility of unmasking existing diabetes rather than new-onset.

The complication of diabetes, such as HHS alone or in combination with DKA, is seen in patients with SARS coV-2 infection. In a case series of six male patients by Chan et al., all patients have an initial presentation of DKA \& HHS together with fatal outcome in four ${ }^{12}$. In a recent review by Pal et al., ${ }^{13} 10 \%$ of the patients have new-onset diabetes mellitus, with $83 \%$ of patients have isolated DKA and $17 \%$ as combined DKA/HHS with male predominance, where mortality is even higher in the latter than the first as $50 \%$.

There are reports of new-onset diabetes mellitus with or without complications in SARS CoV-2 infected individuals. In a retrospective case series of 35 hospitalized hyperglycemic emergencies in SARS CoV-2 infected patients, isolated DKA was (31.4\%), mixed DKA \& HHS (37.1\%), hyperglycemic ketosis (25.7\%), and HHS alone in two African patients (5.7\%). Only two patients were having new-onset diabetes mellitus, one each for DKA \& HHS and HSS alone ${ }^{14}$. 
Un-masking pre-existing diabetes or the development of new-onset diabetes mellitus with accompanying complications like HHS is a possible complication of SARS CoV-2 infection that carries a high mortality. To the best of our knowledge, only one COVID-19 patient with new-onset diabetes mellitus \& HHS, been reported in the published literature. Our case is the second reported case with a complication of HHS in a newly diagnosed diabetes mellitus in a SARS CoV-2 infected patient.

\section{Conclusion :}

Isolated HHS with new-onset diabetes is an unusual but possible complication of SARS CoV-2 infection. SARS CoV-2 infection may trigger $\beta$-cell damage in previously healthy individuals resulting in new-onset diabetes that may present as hyperglycemic hyperosmolar state alone or in combination with ketoacidosis where the outcome may be worst. Such patients should be managed under close observation with established protocols.

\section{Key Clinical Message :}

SARS CoV-2 infection may cause new-onset diabetes mellitus with or without complications such as HHS, which carries the worst prognosis that necessitates specific recommendations and protocols to manage such worst complications in a better way.

\section{Declaration of Competing Interest}

The authors declare that they have no conflict of interest.

\section{Funding}

Nil to declare

\section{Ethical approval}

Not applicable

\section{Author contribution}

Awaji Q. Al-Naami: conceptualization, supervision.

Liaqat A. Khan: conceptualization, methodology, writing, original draft preparation, review \& editing.

Faisal I. Zaidan: Review, and editing.

Ibrahim A. Alneami: supervision, review

Akram S. Awad: Supervision.

Abdulwahab I. Hobani: Supervision, conceptualization

Ali, H. Sheikh: data collection.

Mousa M. Ahmadini: editing, data collection.

\section{References}

1. The Johns Hopkins University of Medicine. COVID-19 dashboard by the Center for Systems Science and Engineering at Johns Hopkins University. https://coronavirus.jhu.edu/map.html Date accessed: November 22, 2020

2. Sanyaolu, A., Okorie, C., Marinkovic, A. et al. Comorbidity and its Impact on Patients with COVID19. SN Compr. Clin. Med. 2, 1069-1076 (2020). https://doi.org/10.1007/s42399-020-00363-4.

3. Hasan Ejaz, Abdullah Alsrhani, Aizza Zafar, Humera Javed, Kashaf Junaid, Abualgasim E. Abdalla, Khalid O.A. Abosalif, Zeeshan Ahmed, Sonia Younas, COVID-19 and comorbidities: Deleterious impact on infected patients, Journal of Infection and Public Health. 2020; [Pre-print] DOI: 10.1016/j.jiph.2020.07.014 https://doi.org/10.1016/j.jiph.2020.07.014. 
4. Zhou F, Yu T, Du R, Fan G, Liu Y, Liu Z, Xiang J, Wang Y, Song B, Gu X, Guan L, Wei Y, Li H, Wu X, Xu J, Tu S, Zhang Y, Chen H, Cao B. Clinical course and risk factors for mortality of adult inpatients with COVID-19 in Wuhan, China: a retrospective cohort study. Lancet 2020;395:1054-62.

5. Yang J-K, Lin S-S, Ji X-J, Guo L-M. Binding of SARS coronavirus to its receptor damages islets and causes acute diabetes. Acta Diabetol 2010 Sep;47:193e9. doi: 10.1007/s00592-009-0109-4

6. Hoffmann, M. et al. SARS-CoV-2 cell entry depends on ACE2 and TMPRSS2 and is blocked by a clinically proven protease inhibitor. Cell2020; 181 (2): 271-280. https://doi.org/10.1016/j.cell.2020.02.052.

7. Fadini, G.P., Morieri, M.L., Longato, E. et al. Prevalence and impact of diabetes among people infected with SARS-CoV-2. J Endocrinol Invest . 2020; 43, 867-869. https://doi.org/10.1007/s40618-02001236-2.

8. Rimesh Pal, Sanjay K. Bhadada, COVID-19 and diabetes mellitus: An unholy interaction of two pandemics, Diabetes \& Metabolic Syndrome: Clinical Research \& Reviews, 2020; 14(4): 513-517 https://doi.org/10.1016/j.dsx.2020.04.049.

9. Hollstein, T., Schulte, D.M., Schulz, J. et al. Autoantibody-negative insulin-dependent diabetes mellitus after SARS-CoV-2 infection: a case report. Nat Metab 2, 1021-1024 (2020). https://doi.org/10.1038/s42255-020-00281-8.

10. Hadil A A O, Eman S, Bashayer Zuhair A S, Anwar J. Diabetic Ketoacidosis and New Onset Diabetes Mellitus Precipitated by COVID-19 Infection. JOJ Case Stud. 2020; 11(3): 555815. DOI 10.19080/JOJCS.2020.11.555815.

11. Thirunavukkarasu Sathish, Yingting Cao, Nitin Kapoor, Newly diagnosed diabetes in COVID 19 patients, Primary Care Diabetes, 10.1016/j.pcd.2020.08.014, (2020). https://doi.org/10.1002/jmv.26339

12. Chan KH, Thimmareddygari D, Ramahi A, Atallah L, Baranetsky NG, Slim J. Clinical characteristics and outcome in patients with combined diabetic ketoacidosis and hyperosmolar hyperglycemic state associated with COVID-19: A retrospective, hospital-based observational case series. Diabetes Res Clin Pract . 2020;166:108279. doi:10.1016/j.diabres.2020.108279.

13. Rimesh Pal, Mainak Banerjee, Urmila Yadav, Sukrita Bhattacharjee, Clinical profile and outcomes in COVID-19 patients with diabetic ketoacidosis: A systematic review of literature, Diabetes \& Metabolic Syndrome: Clinical Research \& Reviews, 2020; 14(6): 1563-1569 https://doi.org/10.1016/j.dsx.2020.08.015.

14. Armeni E, Aziz U, Qamar S, Nasir S, Nethaji C, Negus R, Beynon H.C, Bouloux P, Rosenthal M, Khan $\mathrm{S}$, Yousseif A and Menon. Protracted ketonaemia in hyperglycaemic emergencies in COVID-19: a retrospective case series. Lancet Diabetes Endocrinol . 2020;8(8): 660-663. https://doi.org/10.1016/S22138587(20)30221-7. 